\title{
Comparative analysis of grapevine whole-genome gene predictions, functional annotation, categorization and integration of the predicted gene sequences
}

Jérôme Grimplet ${ }^{1 *}$, John Van Hemert ${ }^{2}$, Pablo Carbonell-Bejerano ${ }^{1,3}$, José Díaz-Riquelme ${ }^{1}$, Julie Dickerson², Anne Fennell ${ }^{4}$, Mario Pezzotti ${ }^{5}$ and José M Martínez-Zapater ${ }^{1,3}$

\begin{abstract}
Background: The first draft assembly and gene prediction of the grapevine genome (8X base coverage) was made available to the scientific community in 2007, and functional annotation was developed on this gene prediction. Since then additional Sanger sequences were added to the $8 \mathrm{X}$ sequences pool and a new version of the genomic sequence with superior base coverage (12X) was produced.

Results: In order to more efficiently annotate the function of the genes predicted in the new assembly, it is important to build on as much of the previous work as possible, by transferring $8 \mathrm{X}$ annotation of the genome to the $12 \mathrm{X}$ version. The $8 \mathrm{X}$ and $12 \mathrm{X}$ assemblies and gene predictions of the grapevine genome were compared to answer the question, "Can we uniquely map $8 \mathrm{X}$ predicted genes to $12 \mathrm{X}$ predicted genes?" The results show that while the assemblies and gene structure predictions are too different to make a complete mapping between them, most genes $(18,725)$ showed a one-to-one relationship between $8 \mathrm{X}$ predicted genes and the last version of $12 \mathrm{X}$ predicted genes. In addition, reshuffled genomic sequence structures appeared. These highlight regions of the genome where the gene predictions need to be taken with caution. Based on the new grapevine gene functional annotation and in-depth functional categorization, twenty eight new molecular networks have been created for VitisNet while the existing networks were updated.

Conclusions: The outcomes of this study provide a functional annotation of the $12 \mathrm{X}$ genes, an update of VitisNet, the system of the grapevine molecular networks, and a new functional categorization of genes. Data are available at the VitisNet website (http://www.sdstate.edu/ps/research/vitis/pathways.cfm).
\end{abstract}

\section{Background}

Due to its substantial economic importance and its position as a model species for perennial fruit crops [1,2], the Vitis genus, particularly the Vitis vinifera species, has benefited from a large effort to develop genomic tools and data [3-6]. Consequently, the bioinformatics resources for the grapevine species has expanded in the past few years, with a variety of tools created for postgenomics era applications [7]. Most notably, the genomes

\footnotetext{
* Correspondence: jerome.grimplet@icvv.es

${ }^{1}$ Instituto de Ciencias de la Vid y del Vino (CSIC, Universidad de La Rioja, Gobierno de La Rioja), CCT, C/Madre de Dios 51, Logroño, España 26006, Vietnam

Full list of author information is available at the end of the article
}

of the heterozygous variety Pinot Noir and a near homozygous Pinot Noir derived inbred (PN40024) have been sequenced $[8,9]$. The sequencing and the assembly of the latter have been updated recently from an $8 \mathrm{X}$ to a $12 \mathrm{X}$ coverage of the genome sequence and a $12 \mathrm{X}$ assembly. The $8 \mathrm{X}$ and $12 \mathrm{X}$ assemblies are accompanied by the respective gene structure predictions, which contain different types of subsequence predictions. These include genes, mRNAs, UTRs, introns, exons, and inter-genic spaces. The methods for gene prediction for the $8 \mathrm{X}$ genomic sequence were previously published [9], using the GAZE software [10]. Two versions of the $12 \mathrm{X}$ prediction are available. Version $0(12 \mathrm{Xv} 0)$ was performed with the GAZE software by the Genoscope in Evry, France. Version
C Biomed Central

(c) 2012 Grimplet et al.; licensee BioMed Central Ltd. This is an Open Access article distributed under the terms of the Creative Commons Attribution License (http://creativecommons.org/licenses/by/2.0), which permits unrestricted use, distribution, and reproduction in any medium, provided the original work is properly cited. 
1 (12Xv1) is the result of the union of $\mathrm{v} 0$ and a gene prediction performed with JIGSAW software [11] at the CRIBI in Padova, Italy [12].

The v0 prediction has been available online since 2009 on the NCBI website and the Genoscope website (www. cns.fr/vitis). The v1 prediction, available at http://genomes. cribi.unipd.it/, was used to design the latest available gene expression microarray for grapevine, based on NimbleGen technology, which is the whole-genome array for grapevine. Other earlier microarray platforms have also been widely popular within the grapevine research community, in particular the two Affymetrix microarray platforms, the Vitis vinifera GeneChip ${ }^{\circledR}$ [13] based on the EST sequences assembled into contigs corresponding to the DFCI gene index version 4 (http://compbio.dfci.harvard. edu/tgi/cgi-bin/tgi/gimain.pl?gudb=grape) and the GrapeGen microarrays [14] based on the DFCI gene index version 5 complemented by the GrapeGen project EST sequences. Within this context, the assessment of the correspondence between all the different sets of grapevine genes ( $8 \mathrm{X}$ and $12 \mathrm{X}$ genome sequence coverage and EST) has several interests: (i) to provide correspondence tables to the grapevine scientific community that relate identical genes with different names, which would allow work performed with the $8 \mathrm{X}$ genome gene IDs to be updated to the $12 \mathrm{X}$ gene IDs; (ii) to use the functional annotation performed on the $8 \mathrm{X}$ predicted genes and to implement it easily on the $12 \mathrm{X}$ genome; (iii) to update VitisNet, which was based on the $8 \mathrm{X}$ prediction [15], for the 12X genome and therefore make it compatible with the NimbleGen array; and (iv) to allow comparison of results obtained from different high throughput platforms such as microarrays or proteomics studies.

\section{Results and discussion}

\section{Sequence homology presents a cardinality problem}

The two available gene predictions of the grapevine genome were produced from an $8 \mathrm{X}$ coverage assembly and from a 12X coverage assembly that included the genomic data from the $8 \mathrm{X}$ coverage version with Arachne [16]. GAZE software was used to perform the gene predictions for the $8 \mathrm{X}$ and the first version of the $12 \mathrm{X}(\mathrm{v} 0)$ prediction. The complete procedure is available in chapter 5 of the supplementary material in Jaillon et al. [9] and was modified to take into account whole transcriptome shotgun data from Solexa sequencing. The procedure for the 12Xv1 is described by Forcato [12]. The v1 gene prediction is the result of the integration between $\mathrm{v} 0$ and the CRIBI prediction that was performed with the JIGSAW software.

The comparison analysis performed by Megablast may result in some genes having a many-to-many relationship between $8 \mathrm{X}$ and $12 \mathrm{X}$ ORFs, with paralogous domains causing a confounding web of links between sets of genes. The degree to which a gene is linked to multiple sister genes in the other assembly versions corresponds to its cardinality with $\mid$ gene $12 \mathrm{X} \mid=$ gene $8 \mathrm{X}_{1}$, gene $\left.8 \mathrm{X}_{\mathrm{n}}\right\}=\mathrm{n}$. To avoid incorrect matching, the chromosome position and matching results for adjacent genes were considered in addition to the sequence similarity.

It has been observed that the $8 \mathrm{X}$ prediction could define a single gene on a specific locus, while a slightly different assembly or prediction method in the $12 \mathrm{X}$ version defined several separate genes spanning the same nucleotides. This results in many $12 \mathrm{X}$ predicted genes aligning almost perfectly with the same $8 \mathrm{X}$ gene. In the case that multiple genes match different portions of a single gene, a possible solution to identify which is the correct prediction is to blast the genes against proteins from other species to observe (i) if the predicted protein either covers multiple similar proteins with different sequences on different regions of its sequence (considered artificial chimeras) or matches a single gene, or (ii) if multiple consecutive genes actually match a single protein or if each one matches a different single protein. Among the genes with cardinality issues, 2363 matched a protein with existence demonstrated at the protein level. After performing this validation, 147 genes from the $12 \mathrm{Xv1}$ prediction seemed to have been wrongly assembled and needed to be split, resulting in a proposed 154 new genes (seven genes were triple chimeras). These genes were identified as "To split" in the Additional file 1 in the column "cardinality between $8 \mathrm{X}$ and $12 \mathrm{X}$ " and in Table 1 . The new genes are identified with " 2 " at the end of the v1

Table 1 Number of predicted gene sequences from the 12Xv1 grapevine genome coverage showing cardinality values higher than one when compared with predicted genes in other versions and assemblies

\begin{tabular}{|c|c|c|c|c|c|c|}
\hline & \multicolumn{3}{|c|}{$\begin{array}{l}\text { Multiple } 12 X v 1 \text { genes matching one } \\
\text { gene in another set }\end{array}$} & \multicolumn{2}{|c|}{$\begin{array}{l}\text { Multiple genes in another set matching } \\
\text { one } 12 X v 1 \text { gene }\end{array}$} & \multirow[t]{2}{*}{$\begin{array}{l}\text { Multiple } \\
\text { situation }\end{array}$} \\
\hline & Redundant & Overlap & Split & Merged & To split & \\
\hline Comparison $8 \mathrm{X}$ & 623 & 1429 & 428 & 1774 & 147 & 122 \\
\hline Comparison mRNA & 54 & 14 & 7 & & & \\
\hline Comparison 12Xv0 & 2735 & & & 846 & & 5 \\
\hline
\end{tabular}

Redundant: multiple genes match the same portion of one gene in other set. Overlap, Split, Merged, and To split: multiple genes match different portions of one gene in other set. Overlap and To split: 12Xv1 genes need to be modified respectively (either merged with another gene or split). 
gene ID in Additional file 1. On the other hand, 1429 gene models seemed to have been incorrectly split in the $12 \mathrm{X}$ and needed to be reassembled. These genes were identified as "Overlap" in Additional file 1 and in Table 1. Also, 1774 genes were correctly merged in the $12 \mathrm{X}$ but split in the $8 \mathrm{X}$ assembly. These genes were identified as "merge" in Additional file 1. Finally, 428 genes were correctly split in $12 \mathrm{X}$ that had been incorrectly merged in the $8 \mathrm{X}$ assembly. These genes were identified as "Split" in Additional file 1 and Table 1. This last set of genes seems to contain a large proportion of genes that were not positioned on known chromosomes in the $8 \mathrm{X}$ assembly and have been placed on chromosomes in the $12 \mathrm{X}$ assembly.

Discrimination of tandemly duplicated genes may be possible with the $12 \mathrm{X}$ coverage. A group of $62312 \mathrm{Xv} 1$ predicted genes matched single genes in the $8 \mathrm{X}$ assembly on the same portion of their respective sequence. These genes were identified as "redundant" in Additional file 1 and Table 1 . Some of these were consecutive $12 \mathrm{Xv} 1$ genes matching a single $8 \mathrm{X}$ gene, most likely corresponding to tandem repeat genes undetected in the $8 \mathrm{X}$ assembly. Another possibility is that two distinct bulks of consecutives genes match a single set of consecutive genes in the $8 \mathrm{X}$, indicating potential mistakes in the $12 \mathrm{X}$ assembly. The possibility that multiple $8 \mathrm{X}$ genes match a single $12 \mathrm{X}$ gene has not been considered since it would most likely correspond to a situation in which the $8 \mathrm{X}$ gene prediction was incorrect. It is less likely that the $12 \mathrm{X}$ gene prediction was incorrect. There were 122 genes from the $12 \mathrm{X}$ assembly that showed a more complex matching pattern involving at least two of the conditions leading to a "many-to-many" relationship between genes. For example there were 61 cases where two $12 \mathrm{X}$ annotated genes $(A$ and $B)$ aligned on different regions of one $8 \mathrm{X}$ gene $(C)$ but another portion of one of them $(B)$ matched to a second $8 \mathrm{X}$ gene $(D)$. As a result, 3 correct answers were possible: keeping gene $A$ and gene $B$ separated (no change); or a portion of gene $B$ needed to be assembled with gene $A$; or gene $A$ and gene $B$ needed to be merged. BLAST analysis was also performed on the non-redundant set of grape mRNAs from the DFCI (Grape gene index $\mathrm{v} 5)$, resulting in fourteen $12 \mathrm{X}$ genes that needed to be merged. For these genes, the comment in Additional file 1 in the cardinality between $8 \mathrm{X}$ and $12 \mathrm{X}$ column is followed by "mRNA".

Similar matching between $12 \mathrm{Xv0}$ and $12 \mathrm{Xv} 1$ assembly versions was also performed. This identified 3581 genes with potential mistakes in structural annotation, thus presenting a degree of cardinality higher than one in 12Xv1. Among these, 1187 genes were identified in the comparison with the $8 \mathrm{X}$.

Most genes did not show cardinality $>1$ and a one-to-one relationship between $12 \mathrm{Xv} 1$ and $8 \mathrm{X}$ could be established for 18,725 genes (Additional file 1). However, 6740 predicted genes from the $12 \mathrm{Xv} 1$ assembly were not found in the $8 \mathrm{X}$ assembly gene prediction, and among them 1342 predicted genes matched transcripts in the EST database (Figure 1). In contrast, 6020 predicted genes in the $8 \mathrm{X}$ gene prediction were not found in the $12 \mathrm{X}$ gene prediction (69 additional genes where only found in the v0). In addition, there were still 11172 non redundant EST sequences from grapevine that did not match any gene from all the public sequenced and assembled grapevine genomes: 5257 from the DFCI v5, 4549 included in the GrapeGen Affymetrix microarray and 1366 from both. Since the $12 \mathrm{Xv} 0$ prediction was considered during the construction of the $12 \mathrm{Xv} 1$, there were only 275 genes from v0 that were not retrieved in v1. They most likely correspond to sequences of low quality that have been filtered out. Additionally, the 3885 genes of the repeat track from the $12 \mathrm{Xv} 1$ were not considered for matching with the $8 \mathrm{X}$ gene prediction although 394 of them matched $12 \mathrm{Xv} 0$ sequences. The repeat track contains highly speculative filtered out (masked) genes, so it was included in this analysis with caution because few of those sequences may be actual genes. The difference between the compositions of the predicted gene sets may be explained by multiple causes. It is reasonable to assume that the $12 \mathrm{X}$ gene prediction is better since it included a higher coverage and an updated gene prediction method from the one used for the $8 \mathrm{X}$ assembly. Therefore, detection of new genes in the $12 \mathrm{X}$ was expected. The absence of almost as many genes from the $8 \mathrm{X}$ prediction is more surprising. It may be related to a better detection of false positives, but there is a possibility that these genes were wrongly undetected in the $12 \mathrm{X}$ gene prediction. For that reason they were kept in the set of unique genes for future analyses.

\section{Relative position of predicted gene sequences}

The relative position of predicted genes in the $8 \mathrm{X}$ and the $12 \mathrm{Xv} 1$ genome coverage sequences was compared as shown in Figure 2 [2]. This figure plots the 23188 unique sister gene pairs. Figures 2A and 2D show the 16997 genes belonging to the same chromosomes in both assemblies. Figure $2 \mathrm{~B}$ shows the 4384 genes that were attributed to the unknown chromosome in at least one assembly. Figure 2C shows the 1807 genes that were allocated onto 2 different chromosomes or were not positioned in a chromosome (random chromosome) between the two assemblies. Sister pairs were coded with colors specific to each chromosome and either stars (odd numbered chromosomes) or circles (even numbered chromosomes) in Figure 2D. Many pairs present a pattern close to $\mathrm{x}=\mathrm{y}$ with only slight deviation (Figure 2D). This shows that sister genes were detected in agreement between both genome versions. 


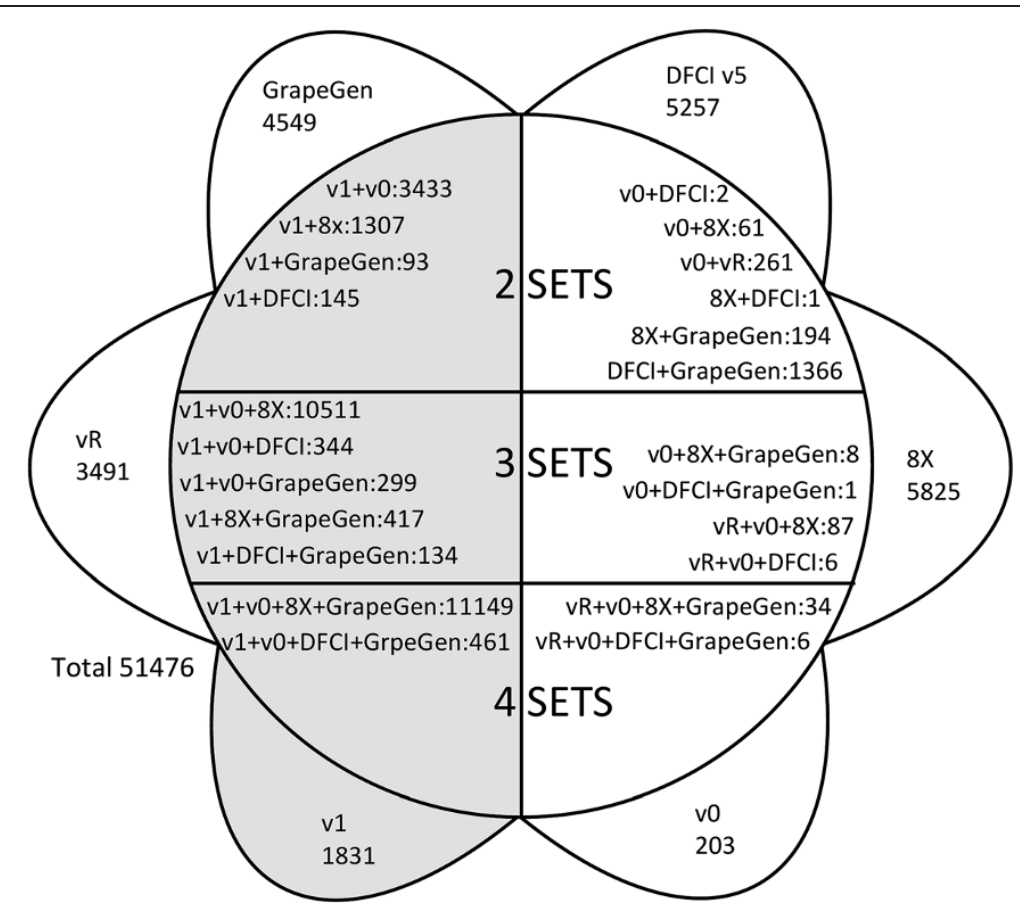

Figure 1 Representation of overlap between the different sets of predicted gene sequences (51476) available for grapevine. 8X: genes identified in the $8 \mathrm{X}$ coverage genome sequence; DFCI v5: mRNA sequences identified in the DFCl gene index EST sequence repository version 5; $\mathrm{V} 1$ : genes identified in the $12 \mathrm{X}$ coverage genome assembly, version 1 of the gene prediction; V0: genes identified in the $12 \mathrm{X}$ coverage genome assembly, version 0 of the gene prediction; VR: predicted genes from the repeat track of the 12X coverage genome sequence, version 1 of the gene prediction; GrapeGen: mRNA sequences identified in the set of mRNA used to construct the GrapeGen Affymetrix microarray; Grey: genes present in the latest update of the protein prediction (12Xv1).

However, important changes between the $8 \mathrm{X}$ and the $12 \mathrm{X}$ assemblies are apparent. These changes can be grouped into four modification types:

i. Genes with unknown chromosomal location in the $8 \mathrm{X}$ that were successfully placed on existing chromosomes in the $12 \mathrm{X}$ (Figure 2B, grey link, 3177 genes). Most of these genes were located inside the first or the last third of the $8 \mathrm{X}$ unknown chromosome sequence. The middle third of the unknown chromosome contains genes that were assigned in the unknown chromosome both in the $8 \mathrm{X}$ and the $12 \mathrm{X}$ assemblies (black links). The middle region contains smaller scaffolds which represents a problem, since increased sequence coverage had no effect on their assignment within chromosomes. In the $8 \mathrm{X}$ assembly unassigned scaffolds were aggregated on the unknown chromosome more or less by size, with the largest towards the edges and the smallest towards the center. It is not surprising that small scaffolds could not originally be assigned to a chromosome as their short length decreases the probability that they will encounter markers linking them to the genetic maps; however, after the $12 \mathrm{X}$ assembly they still were not merged with other scaffolds or their scaffold size did not increase. It is possible that these scaffolds belong to regions difficult to sequence, such as heterochromatin. It has been shown that in Anopheles gambiae, heterochromatin is widely present in unknown chromosome [17]. Syntenic approaches have been used successfully in dog (with human) to clear the ambiguity of unknown chromosome scaffolds, but it is only applicable to scaffolds containing at least 3 genes [18].

ii. In the $8 \mathrm{X}$ assembly some predicted genes were assigned to a given chromosome but were not positioned within it. A significant portion of those predicted genes were definitively located on a chromosomal position (Figure 2C, 1570 genes) in the $12 \mathrm{Xv} 1$ assembly. The most notable sets of newly positioned genes were on chromosomes 1, 15 and 18, with more than 200 genes positioned for each chromosome.

iii. In the $12 \mathrm{X}$ assembly whole chromosome sections were inverted without changing their location (Figure 2D, 1444 genes with a pattern describing a negative correlation between $\mathrm{x}$ and $\mathrm{y}$ ). This is 


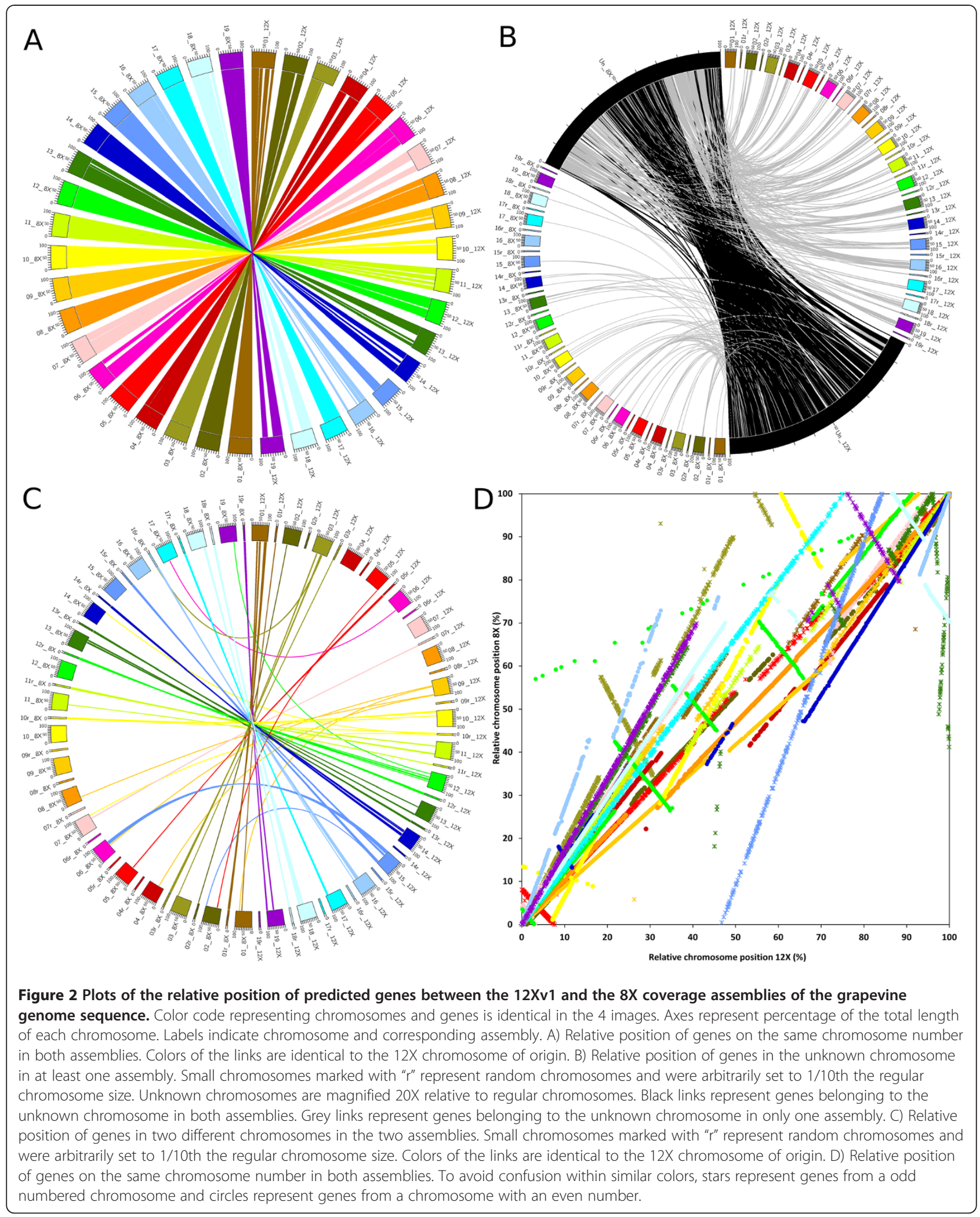

particularly relevant on chromosomes 3 (khaki star, 230 genes), 5 (red star, 174 genes), 7 (pink star, 46 genes) 10 (yellow circle, 45 genes), 11 (light green star, 26 genes) 12 (green circle, 518 genes), 13 (dark green star, 53 genes), 14 (blue circle, 86 genes), 18 (light turquoise circle, 187 genes) and 19 (purple 
circle, 79 genes). Large assembly inversions are most likely caused by sequencing errors on the inversion flanks or ends. These errors could be the result of low coverage in those regions.

iv. One hundred one predicted genes changed chromosomal assignment (Figure 2C). These can be identified by the links describing curves in the figure. A large portion (70 genes) of the $8 \mathrm{X}$ assembly chromosome 6 was placed on chromosome 15 of the $12 \mathrm{X}$ assembly. A smaller group (17 genes) from the random chromosome 16 was definitively attributed to chromosome 3 . An additional 14 genes were assigned to different chromosomes in the two assemblies.

Overall the $12 \mathrm{X}$ assembly shows a clear increase in its accuracy regarding scaffold assignment since a substantial portion of the 8X unknown chromosome and unidentified flanking areas were positioned in the $12 \mathrm{X}$ assembly. Figure $2 \mathrm{~A}$ visualizes the regions that were modified between the assemblies. More specifically, the missing parts in the $12 \mathrm{X}$ chromosomes corresponded to transferred parts visible in Figures $2 \mathrm{C}$ and 2D. The size of the unknown chromosome dropped from $150 \mathrm{Mb}$ in the $8 \mathrm{X}$ assembly to $45 \mathrm{Mb}$ in the $12 \mathrm{X}$ assembly. Relatively few regions have been wrongly assigned to a chromosome in the $8 \mathrm{X}$. Since the $12 \mathrm{X}$ assembly is assumed to be more accurate than the $8 \mathrm{X}$, and few wrongly assigned regions were found between assemblies, the degree of confidence in the scaffold assembly of the $12 \mathrm{X}$ coverage version should be rather high. However, it should be noted that the direction of the assembled scaffold seems to present a high level of discrepancy between the two assemblies, so it is reasonable to assume that accuracy in the $12 \mathrm{X}$ assembly is still not quite optimal.

\section{Functional assignment}

The consensus set of all unique sequences and the sequences unique to any subset were independently compared to sequences from other species and categorized into four main groups (Figure 3): sequences matching another sequence with (i) a known function, (ii) an unknown function, (iii) viral and transposable elements or related sequences and (iv) sequences that do not match any other sequence in public protein databases. Three quarters $(75 \%)$ of the entire set of 12Xv1 genes could be categorized in the first three groups while approximately $15 \%$ did not match anything. In the five other predicted gene subsets, where predictions were only found in one group, the value for group (iv) reached at least $45 \%$. This situation is likely caused by the existence of very short sequences and also explains the difficulties in matching them to the $12 \mathrm{X}$ assembly. In addition, viral and transposable elements represent a relatively high percentage $(>5 \%)$ of the specific predicted genes within the EST and the 8X

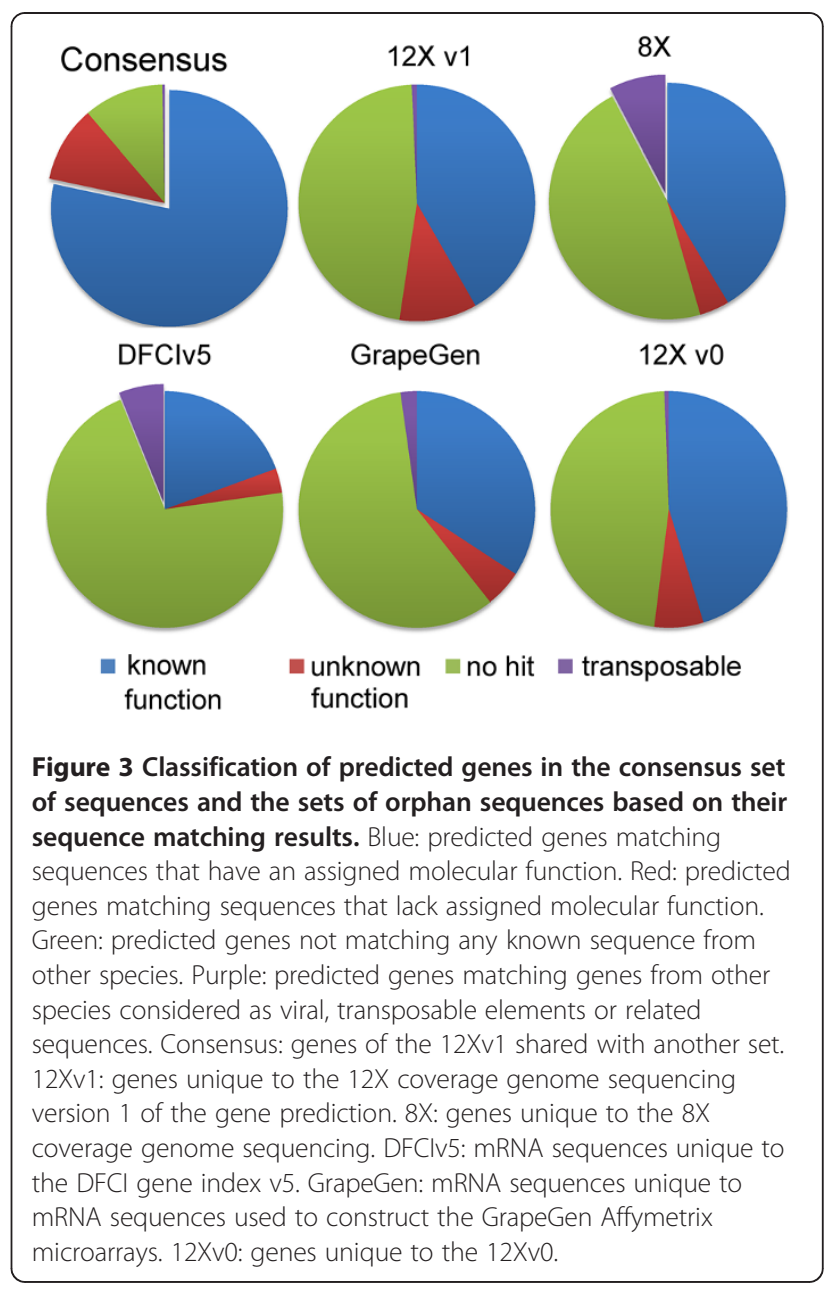

assembly gene prediction that were not retrieved in the $12 \mathrm{X}$ assembly. These genes were filtered from the $12 \mathrm{Xv} 1$ gene prediction, although the repeat track contained a significant proportion of viral sequences. The total consensus set of sequences corresponded to non-redundant sequences in the core set of genes that were already functionally annotated. A majority of these sequences not present in the $12 \mathrm{Xv1}$ may correspond to untranslated or already existing sequences. However, uncertainty remains as to which of these are existing genes but undetected because of short sequence lengths. Match discrepancies in ESTs (DFCI grape gene index v5 and GrapeGen) could also be related in part to varietal specificities.

\section{Functional categorization}

The total 51,476 predicted genes that are potentially unique were assigned to functional categories. For gene categorization, a plant physiology-oriented catalogue was constructed. The catalogue is based on MIPS functional categories, but is complemented by using terms from the GO catalogue that have been hierarchized and fitted 
within the MIPS categories to add a higher level of detail. For example, the MIPS subcategory for heavy metal ion transport lacks resolution. Therefore, subcategories corresponding to GO terms for aluminum, zinc, iron and copper transport were added. Additional attribution of functional categories was performed by converting the VitisNet networks, which frequently corresponded to existing categories, into functional categories. Genes present in a network were considered to belong to the corresponding category. However, transcription factor families in VitisNet corresponding to categories from the transcription factor databases planttfdb [19] or plntfdb [20] are new with regard to MIPS and GO categories. New categories were created and most corresponded to families of genes related to specific plant pathways, particularly those associated with secondary metabolism. The full list of categories and their correspondence with GO and MIPS categories is in Additional file 2. This correspondence allows translation of categories to GO or MIPS. There are 1595 functional categories that contain up to 8 levels of detail. The $12 \mathrm{X}$ predicted genes were allocated to a total of 970 categories. Given the limited experimental information available, most of the unused categories for grapevine genes corresponded to localization (cellular, cell type, tissue and organ).

Figure 4 shows the repartition of the total non-redundant set of predicted genes into higher level functional categories in the primary chart on the left and the repartition within the metabolism category in the secondary chart on the right. The metabolism category is slightly overrepresented when compared to global category values in the literature (closer to 20\%) because a choice was made to include nucleic acid metabolism here instead of placing it into cell fate and DNA processing. Almost half of the genes (44\%) belong to poorly informative categories such as no hit, viral and transposable element, unknown (limited information about the function, presence of a known motif, or known to bind a molecule), unclear (the corresponding protein is somewhat known, but its exact role cannot be determined), or unclassified (the function is not related to any category). Predicted genes belonging to these categories cannot be assigned to lower level categories and would be difficult to place on the VitisNet network without further evidence of their molecular function. One fifth of all the genes $(10,008)$ were present in two or more distinct categories.

\section{VitisNet network update}

VitisNet is a bioinformatics tool that allows the simultaneous integration of "omics" data within grapevine molecular networks [15]. Hence it provides a fast and easy way to monitor the changes in abundance of molecules during a given experiment. Based on the new grapevine genome annotation and the functional sub-categorization of predicted genes, twenty eight new molecular networks have been created for VitisNet. These networks correspond to newly created networks from the KEGG website as well as the transcription factor families recently created on the plant transcription factor databases planttfdb [19] or plntfdb [20]. Among the new networks, two are related to metabolic pathways: glucosinolate biosynthesis and ABA

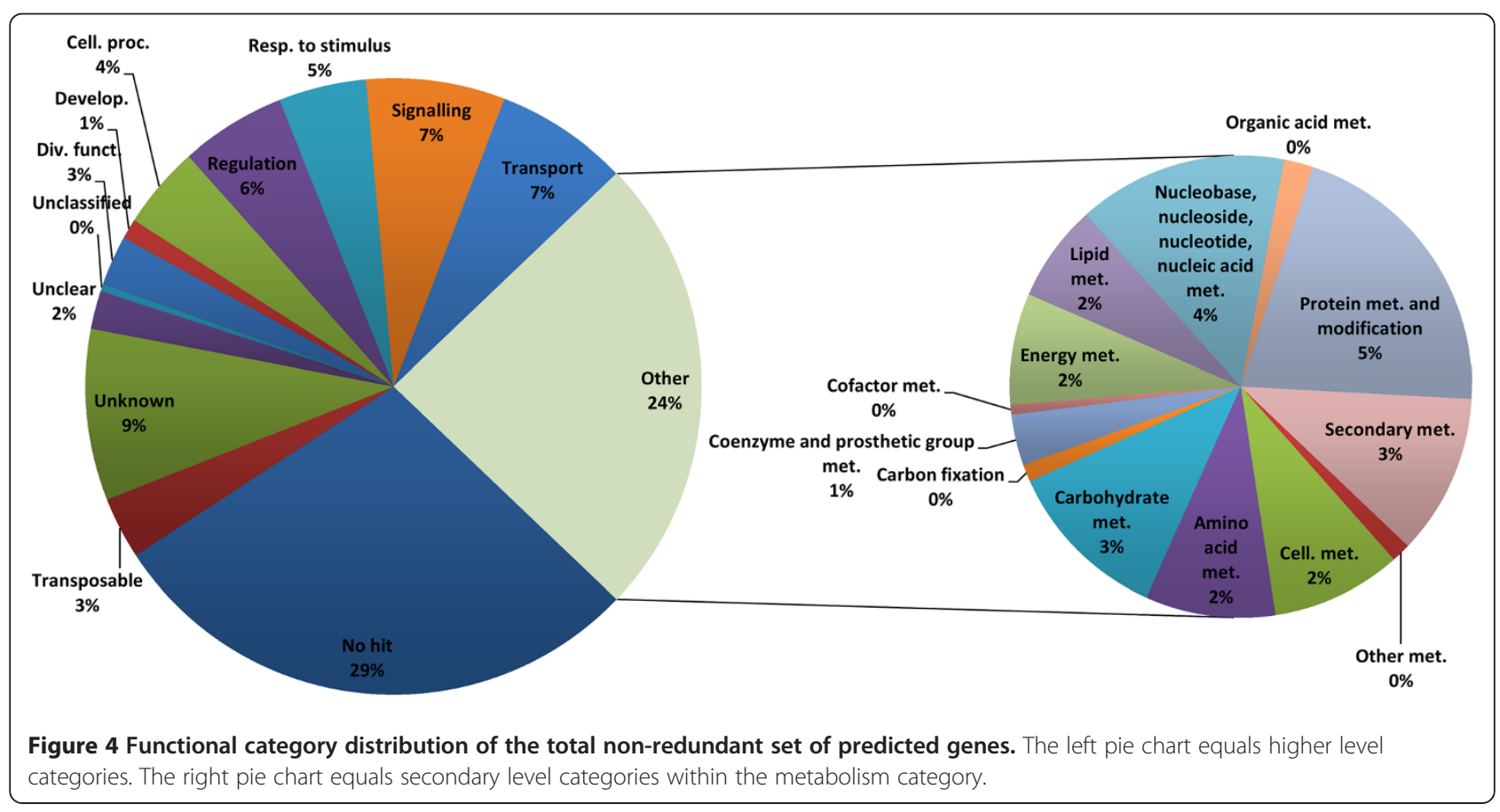


biosynthesis. Seven networks are related to genetic information processing: RNA degradation, spliceosome, ribosome biogenesis in eukaryotes, RNA transport, mRNA surveillance pathway, sulfur relay system, and protein processing in endoplasmic reticulum. Two new networks are related to environmental information processing: plant-pathogen interaction and the $\mathrm{R}$ proteins from plant-pathogen interaction. Two networks are related to cellular processes: peroxisome and phagosome. Finally, fifteen transcription factors families were added: COAP15, GNAT, IWS1, MED, MTERF, OFP, KIP1-SANTA, WRC, nozzle, RCD1, SOH1, SWIB, TRAF, SWI-SNF-SWI3, PAH. Existing networks were also updated with the addition of the predicted genes that are specific to the $12 \mathrm{X}$ as well as genes for which annotation has been revisited. To date, 16,364 (68\%) of the 24155 well characterized genes (belonging to categories other than no hit, transposable element, unclassified, unclear, and unknown) are localized on the molecular networks. The complete list of the networks and numbers of genes, proteins, and metabolites within them are available in Additional file 3.

\section{Conclusions}

The main advantage of developing a unified nomenclature is the ability to port works performed on previous versions of grapevine sequencing projects (both genome and EST). Albeit valuable sources of information, multiplication of grapevine genome sequence sets creates a web of confusion in the analysis of the grapevine genome. The independent development of successive assemblies and gene annotation also results in a loss of information due to the absence of crosschecking between different annotation sources. By studying the differences between the $12 \mathrm{X}$ assembly gene prediction and other gene sets, the work reported here highlights significant discrepancies between the gene predictions from the two genome assemblies. In addition, a significant improvement in the manual functional annotation and categorization of the predicted genes was developed.

\section{Methods}

\section{Predicted genome sequences alignments}

Predicted grapevine sequences corresponding to probable mRNAs were compared in two ways with Megablast [21] between the $8 \mathrm{X}$ and $12 \mathrm{X}$ coverage sets of putative sequences. First, the predicted sequences from the $8 \mathrm{X}$ coverage were the query and the $12 \mathrm{Xv1}$ predicted sequences were the subject database. Second, the predicted sequences from the $12 \mathrm{Xv} 1$ were the query and the $8 \mathrm{X}$ predicted sequences were the subject database. Default Megablast parameters were modified to compare sequences with high stringency, considering only a percentage of identity higher than $95 \%$ and an e-value lower than 1e-20, and displaying only the four best matches. The two BLAST outputs were manually inspected to define the potential true identical sequences according to the results of neighbor genes: at least two consecutively positioned genes from the $12 \mathrm{X}$ version had to hit two consecutive genes in the $8 \mathrm{X}$. Since chromosome sequences have been largely revised, gene positions according to the absolute value within chromosome could not be accurately used to assess the identity between two sequences. Consequently, the relative position between genes was preferentially used.

Cardinality-related discrepancies between the two assemblies identified two groups of genes that required examination (i.e. genes from one assembly presenting a one-to-many relationship with genes from the other assembly). The first group included genes matching multiple counterparts of the other assembly on the same region of their sequence. These might be either tandemly repeated sequences misidentified only once or wrongly duplicated in one assembly, or redundant portions previously assigned to the unknown chromosome. The second group included genes with different regions of their sequence hitting multiple counterparts of the other assembly. The $12 \mathrm{X}$ genes belonging to this group (the one $12 \mathrm{X}$ hitting many $8 \mathrm{X}$ and the many $12 \mathrm{X}$ hitting one $8 \mathrm{X}$ ) were blasted against public protein sequences (Uniprot) to check if two or more sequences corresponded to parts of at least one similar gene from other species. If positive, they were considered unique sequences that needed to be merged. If a sequence hit multiple proteins with different sequences from other species, it was considered as a potential chimera and needed to be split (see the workflow in Additional file 4). Since this step can only be done on one assembly, the genes from the $12 \mathrm{X}$ were preferentially chosen to validate the functional annotation. However, for doubtful situations, the $8 \mathrm{x}$ genes were compared to public databases.

Comparison between v1 and v0 annotations of the $12 \mathrm{X}$ sequence was performed with the same Megablast parameters. However, since the global chromosome sequence structures are identical, gene sequence positions on chromosomes must overlap. Cardinality was also established between the $12 \mathrm{Xv} 1$ and the $12 \mathrm{Xv} 0$ but no check was conducted using other species' proteins as was done in the $8 \mathrm{X}$ to $12 \mathrm{Xv} 1$ comparison.

For the comparison of the 12Xv1 predicted genes with grapevine transcript sequences, hits were considered when homology was higher than $95 \%$ on a length longer than $100 \mathrm{bp}$ and with an e-value $<\mathrm{e}-20$. The $100 \mathrm{bp}$ span criterion was used to compensate for the inability to compare with neighbor genes, as is done in genomic sequence to genomic sequence comparisons. Transcript sequences corresponded on one side to the DFCI Grape gene index v5 and on the other side to the GrapeGen project sequences [22]. EST sequences in these sets are primarily from the cultivars Cabernet Sauvignon (half of the EST 
sequences), Chardonnay, Thompson Seedless, Muscat Hamburg, and Perlette.

\section{Gene annotation}

The total set of genes was built from all genes of the unmodified 12Xv1 prediction complemented with the genes from the other transcript sets and gene predictions that were not identified in $12 \mathrm{Xv1}$. For each gene, the sequence used for comparison with other species and the unique ID was defined according to the following priority order between sets: $12 \mathrm{X}$ sequencing $\mathrm{v} 1>12 \mathrm{X}$ sequencing $\mathrm{v} 0>8 \mathrm{X}$ sequencing $>\mathrm{EST}$ from the DFCI Grape gene index v5 $>$ EST from the GrapeGen project.

Functional annotation was performed, when possible, by inferring the functional annotation of the $8 \mathrm{X}$ genes previously defined [15] to the new set of genes. A de-novo functional characterization was conducted as previously described [15] for genes that were absent from the previous unique gene set. Functional annotation of genes suspected to be involved in newly constructed molecular networks (since the previous release of VitisNet) was reanalyzed even when genes were found in the $8 \mathrm{X}$ prediction. In addition, all the genes presenting cardinality issues were functionally characterized independently of the function assigned in the $8 \mathrm{X}$ set.

\section{VitisNet networks construction}

New networks were constructed since the last update of VitisNet. The new networks were constructed following the protocol described for each network class [15]. The transcription factor networks were completely re-designed since the layout of the transcription factors depends on the phylogenetic relationship of all sequences. Finally, all the $8 \mathrm{X}$-type gene names were replaced by the $12 \mathrm{X}$-type gene IDs according to the latest nomenclature.

\section{Functional categorization}

A functional categories catalog was built by merging categories from the MIPS functional catalog [23] existing in plant species with a selection of GO terms [24] related to plant biological processes and categories related to the VitisNet networks, the transcription factors categories and the TCDB categories [25]. Categories were manually attributed to the genes according to their predicted molecular or physiological function.

\section{Additional files}

Additional file 1: The complete grape gene annotation and correspondence between the sets of sequences. Unique ID: ID from the highest priority level available for a unique gene sequence (priority order $12 X$ sequence $v 1>12 X$ sequence $v 0>8 X$ sequence $>$ EST from DFCl Grape gene index v5 > EST from Grapegen microarrays); gene name followed by an underscore and a number are theoretic genes corresponding to new genes that were incorrectly merged.
Old 12Xv1 name: former name utilized for the $v 1$ of the $12 X$ sequence. 12Xv0 ID: ID from the $v 0$ of the $12 X$ assembly. Identical genes in $8 X$ or other EST: ID of the corresponding gene from the $8 X$ sequencing or EST sequence that does not match an $8 X$ gene. Probeset grapegen: probeset ID for the Affymetrix GrapeGen Vitis vinifera Genome Array. Chromosome position 12X: position of the gene on chromosome in the $12 X$ sequencing assembly; the first part separated by underscore corresponds to the chromosome number, the middle part to the beginning position, and the last part to the end position. Cardinality between $12 X v 0$ and 12Xv1: Comment about the accuracy of the gene prediction inferred from the $v 0$ to $v 1$ comparison; "merge" indicates that multiple sequences of the $v 0$ match one sequence of the $v 1$, "partial" indicates that multiple sequences of the $v 1$ match one sequence of the v0, numbers indicate how may genes from one set match one gene from the other set. Cardinality between $8 X$ and 12Xv1: Comment about the accuracy of the gene prediction inferred from the $8 X$ to $12 X$ comparison; "merge" indicates that multiple sequences of the $8 \mathrm{X}$ assembly match one sequence of the $12 X_{v} 1$ (unless noted otherwise the $12 \mathrm{X}$ assembly gene is correct), "To split" indicates that the $12 \mathrm{X}$ gene is incorrect and needs to be split (if there are more than 2 genes, those that need to be grouped are indicated by order in the column "Identical genes in $8 \mathrm{X}$ or other EST"), "redundant" indicates multiple12X genes matching a single $8 X$ gene on the same position, $X X$ indicates no match between $12 X$ and $8 X, O K$ indicates a one-to-one relationship between $12 \mathrm{X}$ and $8 \mathrm{X}$, "OK (Split)" indicates a 12X gene matching an 8X gene that was an incorrect merging of multiple genes, "Ls" indicates a low score between the matches even though they seem to be correct. Track 12Xv1: the track of the 12Xv1 assembly, either the main track ( 1 1) or the repeat track (V1_r). Functional annotation: tentative functional annotation; briefname, EC or Kegg ID: the identifier that is used in the networks. Network: list of the VitisNet networks in which the gene appears. Functional category: each functional category assigned to the gene. There are up to seven categories for a single gene. Best Arabidopsis match: best matched hit in Arabidopsis putative proteins. Gene Ontology (GO): list of the identified GO terms and their description. Plant Ontology (PO): list of the identified PO terms and their description. Pfam: list of the domains detected from Pfam. Smart: list of the domains detected from Smart. Prosite: list of the domains detected from Prosite. Psort: list of the cellular localization detected from Psort. InterPro domain: list of the domains detected from Interpro. Accession UniProt for published grapevine protein: UniProt ID for grapevine proteins individually published apart from the genome sequencing. Chromosome position $\mathbf{8 X}$ : position of the gene on chromosome in the $8 X$ sequencing assembly. Other Vitis: presence in non-vinifera Vitis species. CDNA array: ID used in the CDNA array from Mathiason et al. (2009). TC from VVGI5: list of other TC from the DFCl matching the gene. GeneChip probesets: probeset ID for the Affymetrix GeneChip ${ }^{\circledR}$ Vitis vinifera (Grape) Genome Array. Best match against proteins with evidence at protein level: compared with uniprot database.

Additional file 2: List of the Vitis putative proteins' functional categories and correspondence with other catalogs. Vitis Functional Category: full name of each functional category. Vitis Functional Category Code: numbered nomenclature of the Vitis Functional Category. Level: hierarchized level of description of the functional category. GO Name: full name of each GO description. GO ID: numbered nomenclature of the GO. VitisNet Network: Corresponding VitisNet network. MIPS Funcat Name: full name of each MIPS functional categories. MIPS Funcat: numbered nomenclature of the MIPS functional category. Number of genes: number of genes belonging to the category.

Additional file 3: List of networks available in VitisNet. VVID VitisNet identification number; gen: number of genes in network; pro: number of proteins in network; $\boldsymbol{m e t}$ : number of metabolites in network. New networks are italicized.

Additional file 4: Analyses workflow for determining cardinality between $8 \mathrm{X}$ and $12 \mathrm{Xv} 1$ assembly genes. Straight line:

representation of the genes from the $12 X_{v} 1$ assembly. Wavy line: representation of the genes from the $8 X$ assembly. Dotted line: genetic sequence. 


\section{Competing interests}

The authors declare that they have no competing interests.

\section{Acknowledgements}

The authors wish to thank Nicola Vitulo and Giorgio Vale for access to preliminary data of the $12 \mathrm{X}$ genome sequencing, Grant Cramer for insights and contribution to VitisNet, and Kathy Mathiason for proofreading the manuscript. This work was supported by project BIO2008-03892 financed by the Spanish MICINN. JG was supported by the JAE Doc program co-funded by the European Social Fund.

\section{Author details}

${ }^{1}$ Instituto de Ciencias de la Vid y del Vino (CSIC, Universidad de La Rioja, Gobierno de La Rioja), CCT, C/Madre de Dios 51, Logroño, España 26006, Vietnam. ${ }^{2}$ Bioinformatics and Computational Biology Department, lowa State University, Ames, IA 50011, USA. ${ }^{3}$ Departamento de Genética Molecular de Plantas, Centro Nacional de Biotecnología, (CNB-CSIC), C/Darwin 3, Madrid, España 28049, Vietnam. ${ }^{4}$ Plant Science Department, South Dakota State University, Brookings, SD 57007, USA. ${ }^{5}$ Department of Biotechnology, University of Verona, Strada le Grazie 15, Verona 37134, Italy.

\section{Authors' contributions}

$J G$ designed the study, performed the analysis, and drafted the manuscript. $\mathrm{JVH}$ and MP participated in the design of the comparison between the $8 \mathrm{X}$ and $12 X$ genome sequences. PCB, JDR and JMMZ participated in the functional assignment and categorization. AF participated in the conception and the update of VitisNet. All authors reviewed the manuscript. All authors read and approved the final manuscript.

Received: 15 December 2011 Accepted: 3 May 2012

Published: 3 May 2012

\section{References}

1. Bouquet A: Grapevines and Viticulture. In Genetics, Genomics, and Breeding of Grapes. Edited by Adam-Blondon A-F, Martinez-Zapater JM, Kole C: Science Publishers; 2011:1-29.

2. Krzywinski M, Schein J, Birol I, Connors J, Gascoyne R, Horsman D, Jones SJ, Marra MA: Circos: an information aesthetic for comparative genomics. Genome Res 2009, 19(9):1639-1645.

3. Adam-Blondon AF, Jaillon O, Vezzulli S, Zharkikh A, Troggio M, Velasco R: Genome Sequence Initiatives. In Genetics, Genomics, and Breeding of Grapes. Edited by Adam-Blondon A-F, Martinez-Zapater JM, Kole C. Enfield: Science Publishers; 2011:211-234.

4. Tillett R, Cushman J: Vitis Functional Genomics: Open Systems for Transcriptome Analysis. In Genetics, Genomics, and Breeding of Grapes. Edited by Adam-Blondon A-F, Martinez-Zapater JM, Kole C. Enfield: Science Publishers; 2011:235-269.

5. Romieu C, Kappel C, Delrot S: Functional Genomics: Closed System Approaches for Transcriptome Analyses. In Genetics, Genomics, and Breeding of Grapes. Edited by Adam-Blondon A-F, Martinez-Zapater JM, Kole C. Enfield: Science Publishers; 2011:270-298.

6. Lund S, Cramer G: Functional Genomics: Proteomics and Metabolomics. In Genetics, Genomics, and Breeding of Grapes. Edited by Adam-Blondon A-F, Martinez-Zapater JM, Kole C. Enfield: Science Publishers; 2011:299-316.

7. Grimplet J, Dickerson J, Adam-Blondon A-F, Cramer G: Bioinformatics Tools in Grapevine Genomics. In Genetics, Genomics, and Breeding of Grapes. Edited by Adam-Blondon A-F, Martinez-Zapater JM, Kole C. Enfield: Science Publishers; 2011:317-331.

8. Velasco R, Zharkikh A, Troggio M, Cartwright DA, Cestaro A, Pruss D, Pindo $M$, Fitzgerald LM, Vezzulli $S$, Reid J, et al: A high quality draft consensus sequence of the genome of a heterozygous grapevine variety. PLOS ONE 2007, 2(12):e1326.

9. Jaillon O, Aury J-M, Noel B, Policriti A, Clepet C, Casagrande A, Choisne N, Aubourg S, Vitulo N, Jubin C, et al: The grapevine genome sequence suggests ancestral hexaploidization in major angiosperm phyla. Nature 2007, 449(7161):463-467

10. Howe KL, Chothia T, Durbin R: GAZE: a generic framework for the integration of gene-prediction data by dynamic programming. Genome Res 2002, 12(9):1418-1427.

11. Allen JE, Salzberg SL: JIGSAW: integration of multiple sources of evidence for gene prediction. Bioinformatics 2005, 21(18):3596-3603.
12. Forcato C: Gene prediction and functional annotation in the Vitis vinifera genome. In PhD Thesis Universita' Degli Studi Di Padova. 2010.

13. Cramer G, Ergul A, Grimplet J, Tillett R, Tattersall E, Bohlman M, Vincent D, Sonderegger J, Evans J, Osborne C, et al: Water and salinity stress in grapevines: early and late changes in transcript and metabolite profiles. Funct Integr Genomics 2007, 7(2):111-134.

14. Pontin MA, Piccoli PN, Francisco R, Bottini R, Martinez-Zapater JM, Lijavetzky $D$ : Transcriptome changes in grapevine (Vitis vinifera L.) cv. Malbec leaves induced by ultraviolet-B radiation. BMC Plant Biol 2010, 10:224.

15. Grimplet J, Cramer GR, Dickerson JA, Mathiason K, Van Hemert J, Fennell AY: VitisNet: "Omics" integration through grapevine molecular networks. PLOS ONE 2009, 4(12):e8365.

16. Jaffe DB, Butler J, Gnerre S, Mauceli E, Lindblad-Toh K, Mesirov JP, Zody MC Lander ES: Whole-genome sequence assembly for mammalian genomes: Arachne 2. Genome Res 2003, 13(1):91-96.

17. Sharakhova MV, George P, Brusentsova IV, Leman SC, Bailey JA, Smith CD Sharakhov IV: Genome mapping and characterization of the Anopheles gambiae heterochromatin. BMC Genomics 2010, 11:459.

18. Derrien T, Andre C, Galibert F, Hitte C: Analysis of the unassembled part of the dog genome sequence: chromosomal localization of 115 genes inferred from multispecies comparative genomics. J Hered 2007, 98(5):461-467.

19. Guo AY, Chen X, Gao G, Zhang H, Zhu QH, Liu XC, Zhong YF, Gu X, He K, Luo J: PlantTFDB: a comprehensive plant transcription factor database. Nucleic Acids Res 2008, 36(suppl_1):D966-D969.

20. Riano-Pachon D, Ruzicic S, Dreyer I, Mueller-Roeber B: PInTFDB: an integrative plant transcription factor database. BMC Bioinformatics 2007, 8:42.

21. Zhang Z, Schwartz S, Wagner L, Miller W: A greedy algorithm for aligning DNA sequences. J Comput Biol 2000, 7(1-2):203-214.

22. Peng FY, Reid KE, Liao N, Schlosser J, Lijavetzky D, Holt R, Martínez Zapater JM, Jones S, Marra M, Bohlmann J, et al: Generation of ESTs in Vitis vinifera wine grape (Cabernet Sauvignon) and table grape (Muscat Hamburg) and discovery of new candidate genes with potential roles in berry development. Gene 2007, 402(1-2):40-50.

23. Ruepp A, Zollner A, Maier D, Albermann K, Hani J, Mokrejs M, Tetko I, Guldener U, Mannhaupt G, Munsterkotter M, et al: The FunCat, a functional annotation scheme for systematic classification of proteins from whole genomes. Nucleic Acids Res 2004, 32(18):5539-5545.

24. Ashburner M, Ball CA, Blake JA, Botstein D, Butler H, Cherry JM, Davis AP, Dolinski K, Dwight SS, Eppig JT, et al: Gene ontology: tool for the unification of biology. The gene ontology consortium. Nat Genet 2000, 25(1):25-29.

25. Saier MH, Tran CV, Barabote RD: TCDB: the transporter classification database for membrane transport protein analyses and information. Nucleic Acids Res 2006, 34(Database issue):D181-D186.

doi:10.1186/1756-0500-5-213

Cite this article as: Grimplet et al: Comparative analysis of grapevine whole-genome gene predictions, functional annotation, categorization and integration of the predicted gene sequences. BMC Research Notes 2012 5:213.

\section{Submit your next manuscript to BioMed Central and take full advantage of:}

- Convenient online submission

- Thorough peer review

- No space constraints or color figure charges

- Immediate publication on acceptance

- Inclusion in PubMed, CAS, Scopus and Google Scholar

- Research which is freely available for redistribution 